\title{
Considering chain-to-chain competition on environmental and social concerns in a supply chain network design problem
}

\author{
Arash Nobari ${ }^{1 *}$, AmirSaman Kheirkhah ${ }^{2}$, Maryam Esmaeili ${ }^{3}$ \\ 1- Industrial Engineering Department, Faculty of Engineering, Bu-Ali Sina University, Hamedan, Iran \\ 2- Industrial Engineering Department, Faculty of Engineering, Bu-Ali Sina University, Hamedan, Iran \\ 3- Industrial Engineering Department, Faculty of Engineering, Alzahra University, Tehran, Iran
}

\begin{abstract}
The supply chain network design problem has been widely studied as one of the most important issues in supply chain management. In recent years, competitive nature of markets caused researchers to attend the competition issue in their studies. In this research, a multi-objective mathematical model for dynamic and integrated network design of a competitive and sustainable supply chain is presented. In the proposed model, a chain-to-chain competition between two supply chains, who compete on environmental and social concerns, is considered. To solve this competitive model, a two-stage solution approach is introduced. A game theory approach is initially used to determine the equilibrium values for competitive decisions. Then, according to results of the competitive stage and respecting complexity of the proposed model, a Pareto-based multi-objective meta-heuristic algorithm is applied to solve the achieved network design problem. Finally, to evaluate the efficiency of the proposed model and presented solution approach, several generated numerical examples are graphically and statistically studied.
\end{abstract}

KEYWORDS: Supply chain network design, chain-to-chain competition, sustainability concerns, game theoretic approach, Pareto-based meta-heuristic algorithm

Kink: https://www.tandfonline.com/doi/full/10.1080/17509653.2018.1474142 